PROCEEDINGS OF THE

AMERICAN MATHEMATICAL SOCIETY

Volume 133, Number 4, Pages 1157-1163

S 0002-9939(04)07665-8

Article electronically published on September 29, 2004

\title{
ON THE EXISTENCE OF PETTIS INTEGRABLE FUNCTIONS WHICH ARE NOT BIRKHOFF INTEGRABLE
}

\author{
JOSÉ RODRÍGUEZ
}

(Communicated by N. Tomczak-Jaegermann)

\begin{abstract}
Let $X$ be a weakly Lindelöf determined Banach space. We prove that if $X$ is non-separable, then there exist a complete probability space $(\Omega, \Sigma, \mu)$ and a bounded Pettis integrable function $f: \Omega \longrightarrow X$ that is not Birkhoff integrable; when the density character of $X$ is greater than or equal to the continuum, then $f$ is defined on $[0,1]$ with the Lebesgue measure. Moreover, in the particular case $X=c_{0}(I)$ (the cardinality of $I$ being greater than or equal to the continuum) the function $f$ can be taken as the pointwise limit of a uniformly bounded sequence of Birkhoff integrable functions, showing that the analogue of Lebesgue's dominated convergence theorem for the Birkhoff integral does not hold in general.
\end{abstract}

\section{INTRODUCTION}

There are several extensions of the theory of Lebesgue integration to the case of functions with values in Banach spaces, those due to Bochner [5] and Pettis [13, 14. [18] being widely known. The Birkhoff integral [1] and the (generalized) McShane integral [8] lie strictly between the Bochner and Pettis integrals and have recently caught the attention of many authors; see [2, 4, 8, 9, 10] and the references therein.

It is well known that if the Banach space in the range is separable, then Pettis, Birkhoff and McShane integrability coincide; see [15 Corollary 5.11] and [8. Corollary $4 \mathrm{C}]$. The differences arise in the non-separable case, and the situation becomes more complicated: every Birkhoff integrable function is McShane integrable, [9, Proposition 4], and every McShane integrable function is Pettis integrable, [8, Theorem 1Q], but none of the reverse implications hold in general, as shown in [9, Example 8] and 10, Example 3C], respectively (more examples can be found in [12, 16 and [4]). However, for certain classes of non-separable Banach spaces some equivalences remain valid: Birkhoff and McShane integrability coincide for functions with values in spaces with weak* separable dual unit ball (equivalently, spaces that are isometric to subspaces of $\ell_{\infty}$ ), see [9, Theorem 10], and McShane and Pettis integrability coincide for functions defined on $[0,1]$ with values in $c_{0}(I)$ ( $I$ any set) or superreflexive spaces; see [4].

Received by the editors December 2, 2003.

2000 Mathematics Subject Classification. Primary 28B05, 46G10; Secondary 46B26.

Key words and phrases. Pettis integral, Birkhoff integral, McShane integral, dominated convergence theorem, Markushevich basis, weakly Lindelöf determined Banach space.

This research was supported by grant BFM2002-01719 of MCYT and FPU grant of MECD (Spain).

(C)2004 American Mathematical Society Reverts to public domain 28 years from publication 
In this paper we try to go a bit further when studying the differences between Birkhoff and Pettis integrability in non-separable Banach spaces. We show that for the wide class of weakly Lindelöf determined Banach spaces (WLD for short, see below for the definition) there is no hope of obtaining a general result on the equivalence of the Pettis and Birkhoff integrals. More precisely, our Theorem 2.1 states that for a non-separable WLD Banach space $X$ there always exist a complete probability space $(\Omega, \Sigma, \mu)$ and a bounded Pettis integrable function $f: \Omega \longrightarrow X$ that is not Birkhoff integrable. For "bigger" spaces this result can be refined in the following way (Theorem 2.3): for a WLD Banach space $X$ with density character greater than or equal to the continuum, there always exists a bounded Pettis integrable function $f:[0,1] \longrightarrow X$ that is not Birkhoff integrable.

In addition, if $I$ is a set of cardinality greater than or equal to the continuum, a particular case of the constructions in Theorem 2.3 gives a uniformly bounded sequence of Birkhoff integrable functions $f_{n}:[0,1] \longrightarrow c_{0}(I)$ that converges pointwise to a function $f:[0,1] \longrightarrow c_{0}(I)$ that is not Birkhoff integrable (Theorem 2.5). This means that the analogue of Lebesgue's dominated convergence theorem for the Birkhoff integral does not hold in general. This negative feature is not shared by the Bochner, McShane and Pettis integrals, for which there are limit theorems that ensure the validity of Lebesgue's theorem (for norm and/or weak convergence); see [5], 8, 10] and 14, respectively.

Our standard references are [7] and [18. Throughout this paper $c$ is the cardinality of the continuum and $\lambda$ stands for the Lebesgue measure on the $\sigma$-algebra $\mathcal{L}$ of Lebesgue measurable subsets of $[0,1]$. The cardinality of an arbitrary set $A$ will be denoted by $|A|$. We write $X^{*}$ to denote the dual of our Banach spaces $X$ (all of them are assumed to be real). $B_{X}$ is the unit ball of $X$. The density character of $X$, denoted by $\operatorname{dens}(X)$, is the minimal cardinality of a norm dense set in $X$. A Markushevich basis of $X$ is a family $\left\{\left(x_{i}, x_{i}^{*}\right)\right\}_{i \in I} \subset X \times X^{*}$ such that (i) $x_{i}^{*}\left(x_{j}\right)=\delta_{i, j}$ (the Kronecker symbol) for every $i, j \in I$; (ii) $\overline{\operatorname{span}}\left\{x_{i}\right\}_{i \in I}=X$ and (iii) for each $x \in X \backslash\{0\}$ there exists $i \in I$ such that $x_{i}^{*}(x) \neq 0$.

Recall that a Banach space $X$ is said to be WLD if $\left(B_{X^{*}}\right.$, weak $\left.{ }^{*}\right)$ is homeomorphic to some subset $S$ of a cube $[-1,1]^{I}$, endowed with the product topology, such that for each $s \in S$ the set $\{i \in I: s(i) \neq 0\}$ is countable. The class of WLD Banach spaces contains all weakly compactly generated spaces (Amir-Lindenstrauss; see [7. Theorem 11.16]) and, more generally, all weakly countably determined ones (see [6, Theorem 7.2.7]). In particular, the spaces considered in [4 for which Pettis and McShane integrability coincide $\left(c_{0}(I)\right.$ and the superreflexive ones) are WLD. The existence of Markushevich bases in WLD Banach spaces, 19, Corollary 3.1] (alternatively see Theorem 12.50 in [7]), will be an essential tool for the proofs of Theorems 2.1 and 2.3

\section{The RESUlts}

Let $(\Omega, \Sigma, \mu)$ be a complete probability space. Recall that a function $f$ defined on $\Omega$ with values in a Banach space $X$ is Birkhoff integrable (with respect to $\mu$ ) if, and only if, for every $\varepsilon>0$ there is a countable partition $\left(B_{n}\right)$ of $\Omega$ in $\Sigma$ such that

$$
\left\|\sum_{n} \mu\left(B_{n}\right) f\left(t_{n}\right)-\sum_{n} \mu\left(B_{n}\right) f\left(t_{n}^{\prime}\right)\right\|<\varepsilon
$$


for arbitrary choices $t_{n}, t_{n}^{\prime} \in B_{n}$, the series involved being unconditionally convergent. Note that the last requirement is automatically fulfilled if $f$ is bounded.

Theorem 2.1. Let $X$ be a WLD Banach space. If $X$ is non-separable, then there exist a complete probability space $(\Omega, \Sigma, \mu)$ and a bounded Pettis integrable function $f: \Omega \longrightarrow X$ that is not Birkhoff integrable.

Proof. As pointed out in the introduction, since $X$ is WLD, there exists a Markushevich basis of $X$, say $\left\{\left(y_{i}, y_{i}^{*}\right)\right\}_{i \in I}$. By [17, Corollary], we can also assume that

$$
\sup _{i \in I}\left\|y_{i}\right\| \cdot\left\|y_{i}^{*}\right\|<\infty
$$

Set $x_{i}=\left\|y_{i}^{*}\right\| \cdot y_{i}$ and $x_{i}^{*}=\left\|y_{i}^{*}\right\|^{-1} \cdot y_{i}^{*}$ for each $i \in I$. Then $\left\{\left(x_{i}, x_{i}^{*}\right)\right\}_{i \in I}$ is a Markushevich basis of $X$ such that $x_{i}^{*} \in B_{X^{*}}$ for every $i \in I$ and $\sup _{i \in I}\left\|x_{i}\right\|<\infty$.

Since $\overline{\operatorname{span}}\left\{x_{i}\right\}_{i \in I}=X$ and $X$ is non-separable, $I$ is uncountable. Set $\Omega:=I$. Let us consider the $\sigma$-algebra $\Sigma$ in $\Omega$ made up of all sets $A \subset \Omega$ for which $A$ or $\Omega \backslash A$ is countable, and take the following complete probability measure $\mu$ on $(\Omega, \Sigma)$ : $\mu(A)=0$ if $A$ is countable, $\mu(A)=1$ otherwise. Define $f: \Omega \longrightarrow X$ by $f(i)=x_{i}$ for every $i \in \Omega$. Obviously $f$ is bounded.

On the one hand, $f$ is not Birkhoff integrable. Our proof is by contradiction. Assume that $f$ is Birkhoff integrable. Then there is a countable partition $\left(B_{n}\right)$ of $\Omega$ in $\Sigma$ such that

$$
\left\|\sum_{n} \mu\left(B_{n}\right) f\left(t_{n}\right)-\sum_{n} \mu\left(B_{n}\right) f\left(t_{n}^{\prime}\right)\right\| \leq \frac{1}{2}
$$

whenever $t_{n}, t_{n}^{\prime} \in B_{n}$. Since $\Omega$ is an atom of $\mu$, all the $B_{n}$ 's but one (say $B_{N}$ ) are of $\mu$-measure 0 . Inequality (1) can now be read as

$$
\begin{aligned}
\frac{1}{2} \geq \sup _{i, j \in B_{N}}\|f(i)-f(j)\| & =\sup _{i, j \in B_{N}}\left\|x_{i}-x_{j}\right\| \\
& \geq \sup _{i, j \in B_{N}} x_{i}^{*}\left(x_{i}-x_{j}\right)=\sup _{i, j \in B_{N}}\left(1-\delta_{i, j}\right),
\end{aligned}
$$

which contradicts the fact that $B_{N}$ has two distinct elements (in fact, $B_{N}$ is uncountable). Therefore, $f$ is not Birkhoff integrable.

On the other hand, $f$ is Pettis integrable. To see this fix $x^{*} \in X^{*}$. Since $X$ is WLD, the set $\left\{i \in I: x^{*}\left(x_{i}\right) \neq 0\right\}$ is countable, see [7. Proposition 12.51], and therefore $x^{*} f$ vanishes $\mu$-almost everywhere. Since $x^{*} \in X^{*}$ is arbitrary, $f$ is scalarly $\mu$-null and, in particular, Pettis integrable. The proof is complete.

It follows that a WLD Banach space $X$ is separable if, and only if, every (bounded) Pettis integrable function defined on a complete probability space with values in $X$ is Birkhoff integrable. Therefore, whereas the coincidence of Pettis and Bochner integrability characterizes the finite-dimensional Banach spaces, the coincidence of Pettis and Birkhoff integrability characterizes the separable spaces inside the class of WLD Banach spaces.

Our proof of Theorem 2.3 below is inspired by some ideas in the example of a Pettis integrable function that is not Birkhoff integrable given in [12]. We first need the result isolated in Lemma [2.2. Observe that, since the Borel $\sigma$-algebra of $[0,1]$ has cardinality $c$, the same also holds for the collection of all countable partitions of $[0,1]$ by Borel sets, which we enumerate as $\left\{\Gamma_{\alpha}: \alpha<c\right\}$. 
Lemma 2.2. There exist collections $\left\{A_{\alpha}\right\}_{\alpha<c}$ and $\left\{A_{\alpha}^{\prime}\right\}_{\alpha<c}$ of countable subsets of $[0,1]$ such that

(i) $A_{\alpha} \cap A_{\beta}=\emptyset$ and $A_{\alpha}^{\prime} \cap A_{\beta}^{\prime}=\emptyset$ for every $\alpha, \beta<c$ with $\alpha \neq \beta$;

(ii) $A_{\alpha} \cap A_{\beta}^{\prime}=\emptyset$ for every $\alpha, \beta<c$;

(iii) $A_{\alpha} \cap E \neq \emptyset$ and $A_{\alpha}^{\prime} \cap E \neq \emptyset$ for every $\alpha<c$ and every $E \in \Gamma_{\alpha}$ with $\lambda(E)>0$.

Proof. We proceed by transfinite induction. The first step is easy, since $A_{0}$ and $A_{0}^{\prime}$ can be obtained by choosing exactly one point of each member of $\Gamma_{0}$ of positive $\lambda$-measure (taking different points for $A_{0}$ and $A_{0}^{\prime}$, of course). Suppose now that $\alpha<c$ and that we have already constructed two collections $\left\{A_{\beta}\right\}_{\beta<\alpha}$ and $\left\{A_{\beta}^{\prime}\right\}_{\beta<\alpha}$ of countable subsets of $[0,1]$ with the following properties: (1) $A_{\beta} \cap A_{\gamma}=\emptyset$ and $A_{\beta}^{\prime} \cap A_{\gamma}^{\prime}=\emptyset$ for every $\beta, \gamma<\alpha$ with $\beta \neq \gamma$; (2) $A_{\beta} \cap A_{\gamma}^{\prime}=\emptyset$ for every $\beta, \gamma<\alpha$; (3) $A_{\beta} \cap E \neq \emptyset$ and $A_{\beta}^{\prime} \cap E \neq \emptyset$ for every $\beta<\alpha$ and every $E \in \Gamma_{\beta}$ with $\lambda(E)>0$.

Observe that $A:=\bigcup_{\beta<\alpha}\left(A_{\beta} \cup A_{\beta}^{\prime}\right)$ has cardinality $|A|<c$, because each $A_{\beta} \cup$ $A_{\beta}^{\prime}$ is countable and $|\alpha|<c$. Since each uncountable Borel subset of $[0,1]$ has cardinality $c$, see [3, Theorem 8.3.6], it follows that if $E \in \Gamma_{\alpha}$ and $\lambda(E)>0$, then $|E \backslash A|=c$ and, in particular, $E \backslash A$ has two distinct elements. Therefore, $A_{\alpha}$ and $A_{\alpha}^{\prime}$ can be constructed by choosing exactly one element of $E \backslash A$ for every $E \in \Gamma_{\alpha}$ of positive $\lambda$-measure (with different choices for $A_{\alpha}$ and $A_{\alpha}^{\prime}$ ). The proof is finished.

Theorem 2.3. Let $X$ be a $W L D$ Banach space. If $\operatorname{dens}(X) \geq c$, then there exists a bounded Pettis integrable function $f:[0,1] \longrightarrow X$ that is not Birkhoff integrable.

Proof. As in the proof of Theorem 2.1, $X$ admits a Markushevich basis $\left\{\left(x_{i}, x_{i}^{*}\right)\right\}_{i \in I}$ such that $x_{i}^{*} \in B_{X^{*}}$ for every $i \in I$ and $\sup _{i \in I}\left\|x_{i}\right\|<\infty$.

Since $\operatorname{span}\left\{x_{i}\right\}_{i \in I}=X$ and $\operatorname{dens}(X) \geq c$, the cardinality of $I$ is greater than or equal to $c$. Fix two injective maps $\phi, \psi: c \longrightarrow I$ such that $\phi(c) \cap \psi(c)=\emptyset$. Let $\left\{A_{\alpha}\right\}_{\alpha<c}$ and $\left\{A_{\alpha}^{\prime}\right\}_{\alpha<c}$ be the collections of subsets of $[0,1]$ obtained in Lemma 2.2 Define $f:[0,1] \longrightarrow X$ by

$$
f(t)= \begin{cases}x_{\phi(\alpha)} & \text { if } t \in A_{\alpha}, \alpha<c, \\ x_{\psi(\alpha)} & \text { if } t \in A_{\alpha}^{\prime}, \alpha<c, \\ 0 & \text { if } t \notin \bigcup_{\alpha<c}\left(A_{\alpha} \cup A_{\alpha}^{\prime}\right) .\end{cases}
$$

Next we show that the bounded function $f$ satisfies the required properties.

To see that $f$ is Pettis integrable fix $x^{*} \in X^{*}$. We already know that the set $\left\{i \in I: x^{*}\left(x_{i}\right) \neq 0\right\}$ is countable. Since $\phi$ and $\psi$ are injective and the $A_{\alpha}$ 's and $A_{\alpha}^{\prime}$ 's are countable, the set $\left\{t \in[0,1]: x^{*} f(t) \neq 0\right\}$ is countable. Since $x^{*} \in X^{*}$ is arbitrary, $f$ is scalarly $\lambda$-null and, therefore, Pettis integrable.

To finish the proof we will show that $f$ is not Birkhoff integrable by contradiction. Assume that $f$ is Birkhoff integrable. Then there exists a countable partition $\Gamma=\left(B_{n}\right)$ of $[0,1]$ in $\mathcal{L}$ such that

$$
\left\|\sum_{n} \lambda\left(B_{n}\right) f\left(t_{n}\right)-\sum_{n} \lambda\left(B_{n}\right) f\left(t_{n}^{\prime}\right)\right\| \leq \frac{1}{2}
$$

for arbitrary choices $t_{n}, t_{n}^{\prime} \in B_{n}$. By the inner regularity of $\lambda$ with respect to the Borel $\sigma$-algebra of $[0,1]$, we can suppose without loss of generality that $\Gamma$ is made up of Borel sets, that is, $\Gamma=\Gamma_{\alpha}$ for some $\alpha<c$. 
Since $A_{\alpha} \cap B_{n} \neq \emptyset$ and $A_{\alpha}^{\prime} \cap B_{n} \neq \emptyset$ whenever $\lambda\left(B_{n}\right)>0$, for each $n \in \mathbb{N}$ there are some $t_{n}, t_{n}^{\prime} \in B_{n}$ such that $\sum_{n} \lambda\left(B_{n}\right) f\left(t_{n}\right)=x_{\phi(\alpha)}$ and $\sum_{n} \lambda\left(B_{n}\right) f\left(t_{n}^{\prime}\right)=x_{\psi(\alpha)}$. It follows that

$$
\begin{aligned}
\left\|\sum_{n} \lambda\left(B_{n}\right) f\left(t_{n}\right)-\sum_{n} \lambda\left(B_{n}\right) f\left(t_{n}^{\prime}\right)\right\| & =\left\|x_{\phi(\alpha)}-x_{\psi(\alpha)}\right\| \\
& \geq x_{\phi(\alpha)}^{*}\left(x_{\phi(\alpha)}-x_{\psi(\alpha)}\right)=1,
\end{aligned}
$$

which contradicts (3) and shows that $f$ is not Birkhoff integrable. The proof is finished.

Observe that the function $f$ constructed in the proof of Theorem 2.3 is even universally Pettis integrable, i.e., $f$ is Pettis integrable with respect to each Radon measure on $[0,1]$. This follows immediately from the boundedness of $f$ and the fact that $\left\{t \in[0,1]: x^{*} f(t) \neq 0\right\}$ is countable for every $x^{*} \in X^{*}$.

As a consequence of Theorem 2.3 it turns out that, under the Continuum Hypothesis, a WLD Banach space $X$ is separable if, and only if, every (bounded) Pettis integrable function $f:[0,1] \longrightarrow X$ is Birkhoff integrable.

Corollary 2.4. Let $X$ be a superreflexive Banach space. If $\operatorname{dens}(X) \geq c$, then there exists a bounded McShane integrable function $f:[0,1] \longrightarrow X$ that is not Birkhoff integrable.

Proof. Since $X$ superreflexive, Pettis and McShane integrability coincide for functions $f:[0,1] \longrightarrow X$; see 4 . The result now follows from Theorem 2.3

From now on, given a set $I,\left\{\left(x_{i}, x_{i}^{*}\right)\right\}_{i \in I}$ will denote the standard Markushevich basis of $c_{0}(I)$ (i.e. $x_{i}(j)=\delta_{i, j}$ for every $i, j \in I$ and $x_{i}^{*}(y)=y(i)$ for every $y \in c_{0}(I)$ and every $i \in I)$.

Theorem 2.5. Let $I$ be a set of cardinality $|I| \geq c$. Then there exists a uniformly bounded sequence $f_{n}:[0,1] \longrightarrow c_{0}(I)$ of Birkhoff integrable functions that converges pointwise to a function $f:[0,1] \longrightarrow c_{0}(I)$ that is not Birkhoff integrable.

Proof. Let $f:[0,1] \longrightarrow c_{0}(I)$ be the function given by (2) associated to the basis $\left\{\left(x_{i}, x_{i}^{*}\right)\right\}_{i \in I}$. The proof of Theorem 2.3 reveals that $f$ is not Birkhoff integrable.

Write $A_{\alpha}=\left\{a_{\alpha, 1}, a_{\alpha, 2}, \ldots\right\}$ and $A_{\alpha}^{\prime}=\left\{a_{\alpha, 1}^{\prime}, a_{\alpha, 2}^{\prime}, \ldots\right\}$ for every $\alpha<c$, and define $D_{n}:=\left\{a_{\alpha, n}: \alpha<c\right\} \cup\left\{a_{\alpha, n}^{\prime}: \alpha<c\right\}$ and $E_{n}:=\bigcup_{k=1}^{n} D_{k}$ for every $n \in \mathbb{N}$. Define $h_{n}:=f \chi_{D_{n}}$ and $f_{n}:=f \chi_{E_{n}}=\sum_{k=1}^{n} h_{k}$ for every $n \in \mathbb{N}$ (where $\chi_{A}$ denotes the characteristic function of the set $A)$. Then $\left(f_{n}\right)$ is a uniformly bounded sequence (since $f$ is bounded) that converges pointwise to $f$. In order to prove that each $f_{n}$ is Birkhoff integrable it suffices to check that each $h_{n}$ is Birkhoff integrable. To this end fix $n \in \mathbb{N}$ and $\varepsilon>0$. Choose a finite partition $\left(C_{k}\right)_{1 \leq k \leq m}$ of $[0,1]$ in $\mathcal{L}$ such that $\lambda\left(C_{k}\right) \leq \varepsilon$ for every $1 \leq k \leq m$. Observe that if $t_{k} \in C_{k}$ for every $1 \leq k \leq m$, then

$$
\left\|\sum_{k=1}^{m} \lambda\left(C_{k}\right) h_{n}\left(t_{k}\right)\right\|_{\infty} \leq \max _{1 \leq k \leq m} \lambda\left(C_{k}\right) \leq \varepsilon,
$$

since $\left.h_{n}\right|_{D_{n}}=\left.f\right|_{D_{n}}$ is injective. Since $\varepsilon>0$ is arbitrary, $h_{n}$ is Birkhoff integrable. It follows that $f_{n}$ is Birkhoff integrable for every $n \in \mathbb{N}$, and the proof is finished. 
Observe that the functions $f_{n}$ obtained in the proof of Theorem 2.5 are even Riemann integrable, i.e., given $n \in \mathbb{N}$, for every $\varepsilon>0$ there is a finite family $\left(I_{k}\right)$ of non-overlapping closed intervals covering $[0,1]$ such that

$$
\left\|\sum_{k} \lambda\left(I_{k}\right) f_{n}\left(t_{k}\right)-\sum_{k} \lambda\left(I_{k}\right) f_{n}\left(t_{k}^{\prime}\right)\right\|_{\infty}<\varepsilon
$$

whenever $t_{k}, t_{k}^{\prime} \in I_{k}$. For a detailed account of the theory of Riemann integration in Banach spaces we refer the reader to [11].

We finish the paper with Corollary [2.6] below (already known; see [9, Example 8]). Although this result can be deduced in the same way as we did in Corollary [2.4 (recall that Pettis and McShane integrability coincide for functions defined on $[0,1]$ with values in $c_{0}(I)$, (4), the particular properties of our constructions allow us to provide a direct proof.

Corollary 2.6. Let $I$ be a set with cardinality $|I| \geq c$. Then there exists a bounded McShane integrable function $f:[0,1] \longrightarrow c_{0}(I)$ that is not Birkhoff integrable.

Proof. We use the terminology of [10]. Let $f:[0,1] \longrightarrow c_{0}(I)$ be the function constructed in the proof of Theorem 2.5. We know that $f$ is not Birkhoff integrable. In order to prove that $f$ is McShane integrable fix $\varepsilon>0$. For each $\alpha<c$ the set $A_{\alpha}$ (resp. $\left.A_{\alpha}^{\prime}\right)$ is countable, and we can choose an open set $A_{\alpha} \subset G_{\alpha} \subset \mathbb{R}$ (resp. $\left.A_{\alpha}^{\prime} \subset G_{\alpha}^{\prime} \subset \mathbb{R}\right)$ such that $\lambda\left(G_{\alpha}\right) \leq \varepsilon\left(\right.$ resp. $\lambda\left(G_{\alpha}^{\prime}\right) \leq \varepsilon$ ). Fix any gauge $\delta:[0,1] \longrightarrow \mathbb{R}^{+}$such that $[t-\delta(t), t+\delta(t)] \subset G_{\alpha}\left(\operatorname{resp} .[t-\delta(t), t+\delta(t)] \subset G_{\alpha}^{\prime}\right)$ whenever $t \in A_{\alpha}$ (resp. $t \in A_{\alpha}^{\prime}$ ) and $\alpha<c$.

Now, if $\left\langle\left(\left[a_{i}, b_{i}\right], t_{i}\right)\right\rangle_{1 \leq i \leq n}$ is a McShane partition of $[0,1]$ subordinate to $\delta$, we have

$$
\begin{aligned}
\| \sum_{i=1}^{n}\left(b_{i}-a_{i}\right) f\left(t_{i}\right) & \|_{\infty} \\
= & \left\|\sum_{\alpha<c} \lambda\left(\bigcup_{t_{i} \in A_{\alpha}}\left[a_{i}, b_{i}\right]\right) x_{\phi(\alpha)}+\sum_{\alpha<c} \lambda\left(\bigcup_{t_{i} \in A_{\alpha}^{\prime}}\left[a_{i}, b_{i}\right]\right) x_{\psi(\alpha)}\right\|_{\infty} \leq \varepsilon,
\end{aligned}
$$

since $\bigcup_{t_{i} \in A_{\alpha}}\left[a_{i}, b_{i}\right] \subset G_{\alpha}$ and $\bigcup_{t_{i} \in A_{\alpha}^{\prime}}\left[a_{i}, b_{i}\right] \subset G_{\alpha}^{\prime}$ for every $\alpha<c$. Since $\varepsilon>0$ is arbitrary, $f$ is McShane integrable, with integral 0 , and the proof is complete.

\section{REFERENCES}

1. G. Birkhoff, Integration of functions with values in a Banach space, Trans. Amer. Math. Soc. 38 (1935), no. 2, 357-378. MR1501815

2. B. Cascales and J. Rodríguez, The Birkhoff integral and the property of Bourgain, To appear in Math. Ann.

3. D. L. Cohn, Measure theory, Birkhäuser Boston Inc., Boston, MA, 1993, Reprint of the 1980 original. MR1454121 (98b:28001)

4. L. Di Piazza and D. Preiss, When do McShane and Pettis integrals coincide?, Illinois J. Math. 47 (2003), 1177-1187. MR.2036997

5. J. Diestel and J. J. Uhl, Jr., Vector measures, American Mathematical Society, Providence, R.I., 1977, With a foreword by B. J. Pettis, Mathematical Surveys, No. 15. MR0453964 $(56: 12216)$

6. M. Fabian, Gâteaux differentiability of convex functions and topology, Canadian Mathematical Society Series of Monographs and Advanced Texts, John Wiley \& Sons Inc., New York, 1997, Weak Asplund spaces, A Wiley-Interscience Publication. MR 1461271 (98h:46009)

7. M. Fabian, P. Habala, P. Hájek, V. Montesinos Santalucia, J. Pelant, and V. Zizler, Functional analysis and infinite-dimensional geometry, CMS Books in Mathematics/Ouvrages 
de Mathématiques de la SMC, vol. 8, Springer-Verlag, New York, 2001. MR1831176 (2002f:46001)

8. D. H. Fremlin, The generalized McShane integral, Illinois J. Math. 39 (1995), no. 1, 39-67. MR1299648 (95j:28008)

9. Mathematics Department Research Report 92-10, 1999.

10. D. H. Fremlin and J. Mendoza, On the integration of vector-valued functions, Illinois J. Math. 38 (1994), no. 1, 127-147. MR.1245838(94k:46083)

11. R. A. Gordon, Riemann integration in Banach spaces, Rocky Mountain J. Math. 21 (1991), no. 3, 923-949. MR 1138145 (92k:28017)

12. V. M. Kadets and L. M. Tseytlin, On "integration" of non-integrable vector-valued functions, Mat. Fiz. Anal. Geom. 7 (2000), no. 1, 49-65. MR1760946|(2001e:28017)

13. K. Musial, Topics in the theory of Pettis integration, Rend. Istit. Mat. Univ. Trieste 23 (1991), no. 1, 177-262 (1993), School on Measure Theory and Real Analysis (Grado, 1991). MR $1248654(94 \mathrm{k}: 46084)$

14. 2002, pp. 531-586. MR.1954622(2004d:28026)

15. B. J. Pettis, On integration in vector spaces, Trans. Amer. Math. Soc. 44 (1938), no. 2, 277-304. MR1501970

16. R. S. Phillips, Integration in a convex linear topological space, Trans. Amer. Math. Soc. 47 (1940), 114-145. MR.0002707 (2:103c)

17. A. N. Plichko, On projective resolutions of the identity operator and Markushevich bases, Soviet Math. Dokl. 25 (1982), no. 2, 386-389.

18. M. Talagrand, Pettis integral and measure theory, Mem. Amer. Math. Soc. 51 (1984), no. 307, $\mathrm{ix}+224$. MR $0756174 \mid(86 \mathrm{j}: 46042)$

19. M. Valdivia, Simultaneous resolutions of the identity operator in normed spaces, Collect. Math. 42 (1991), no. 3, 265-284 (1992). MR1203185 (94e:46047)

Departamento de Matemáticas, Universidad de Murcia, 30.100 Espinardo, Murcia, SPAIN

E-mail address: joserr@um.es 